\title{
ASME2017/63573
}

\section{CFD SIMULATION OF BLADE FLOWS WITH HIGH AMPLITUDE PITCHING}

\author{
Ramesh Kumar ${ }^{*}$, John W. Chew ${ }^{*}$, Dario Amirante*, Joseba Murua ${ }^{* *}$ and Nicholas J. Hills ${ }^{*}$ \\ *Rolls-Royce Thermo Fluid-System UTC, University of Surrey, Guildford, UK \\ ** Pilatus Aircraft Ltd,P.O. Box 992, 6371 Stans, Switzerland
}

\begin{abstract}
Large and flexible wind turbine blades may be susceptible to severe blade deformations coupled with dynamic stall. To advance prediction capability for this problem a general deforming mesh computational fluid dynamics (CFD) method has been developed for calculating flows with moving or deforming boundaries using an elastic spring analogy. The method has been evaluated against experimental data for flow around a pitching NACA0012 airfoil in the deep dynamic stall regime where flow is highly separated, and compared with other authors' CFD simulations for pitching airfoil. The effects of varying the reduced frequency are also investigated. During the upstroke the present results are in generally good agreement with experiment and other CFD studies. During the downstroke some differences with experiment and other CFD models are apparent. This may be due to the sensitivity of the separated flow to modelling assumptions and experimental conditions. Overall, the degree of agreement between CFD and experiment is considered encouraging.
\end{abstract}

Keywords: CFD, Dynamic Stall, LEV, TEV

\section{NOMENCLATURE}

c airfoil chord

$C_{d}$ sectional drag coefficient

$C_{l}$ sectional lift coefficient

$C_{p}$ pressure coefficient

$\kappa$ reduced frequency, $\omega \mathrm{c} / 2 V_{\infty}$

M mach number

$\mathrm{P}$ pressure

Re Reynolds number

$\mathrm{t}$ time

\footnotetext{
*All corresponds to ramesh.kumar@surrey.ac.uk
}

\author{
$V_{\infty} \quad$ free stream velocity \\ $Y^{+}$non-dimensional near wall mesh spacing \\ $\alpha$ angle of attack \\ $\alpha_{o} \quad$ mean angle of attack \\ $\alpha_{a}$ oscillation amplitude \\ $\omega$ circular frequency \\ $\tau$ stress tensor
}

\section{INTRODUCTION}

Dynamic stall is a complex phenomenon in which a shear layer rolls up and forms a leading edge vortex (LEV) which becomes unstable after a certain flow time. During dynamic stall the onset of stall occurs much later than in static stall, and the unsteady loads generated can be large. During the late $70^{\prime} \mathrm{s} \mathrm{Mc}$ Croskey et al. [1] conducted an experimental study on a pitching NACA0012 airfoil and found that the turbulent vortex breakdown is a key element of dynamics stall. Mehta et al. [2] performed experimental analysis on the flow pattern over a pitching airfoil. Later on, numerous investigations were conducted including study of the effects of profile shape and compressibility [3, 4, 5, 6]. There is also a significant amount of published research on the effect of free stream turbulent intensity, Mach number, Reynolds number and the reduced frequency [7, 8, 9]. Researchers all around the world have analysed the flow over an airfoil in the dynamic stall motivated by application in helicopter blade and micro air vehicle (MAV) aerodynamics.

Leishman et al. [10] has classified dynamic stall into three types of stall namely light, moderate and deep dynamic stall, depending on reduced frequency. Dynamic stall studies can also be separated into low Reynolds number $\left(<10^{5}\right)$ and high Reynolds number $\left(\operatorname{Re}>10^{6}\right)$ investigations. In MAVs pitching and flaping are associated with low Reynolds number. Sarkar et al. [11] have studied the evolution and growth of leading and trailing edge stall
Copyright (C) 2017 by ASME 
vortex over a sinusoidal pitching airfoil at low Re. The effect of mean pitching angle and the amplitude of the pitching angle also studied at low Reynolds number by Akbari et al. [12]. Further investigations in the low Reynolds number regime are reported by Kang et al. [13], Rival and Tropea [14], and Wernet et al. [15].

Recently wind energy researchers have been put significant effort into improved aerodynamic and structural modeling. Wernet et al. [7] performed the CFD simulation for vertical axis wind turbine blade section at low Reynolds number $\left(10^{5}\right)$ Martinat et al. [16] performed a CFD simulation for pitching airfoil and compared the results with experimental results of McCroskey et al. [17]. This investigation was performed at a Reynolds number of 1 million which falls in the operating range of wind turbines. It was found that the stalling angle and formation of leading edge vortices depends on the reduced frequency of the oscillations. McCroskey et al. [1] categorized the formation of primary LEV, secondary LEV and their shedding into three types. At higher reduced frequencies, the separation of primary LEV and propagation and separation of a secondary LEV take place in the downstroke. for moderate reduced frequency, the shedding of primary LEV could takes place in the upstroke and for lower reduced frequency the shedding of secondary vortex also could be observed in the upstroke.

Aerodynamic load prediction in the event of dynamic stall remains challenging due to the large separation taking place over the blade surface. Here load and stalling angle prediction by a current numerical approach is reported and compared with available experimental and CFD simulation results of other authors ${ }^{\prime}$ for the a high Reynolds number test case. The effect of reduced frequency is also investigated using CFD simulation.

\section{CFD modeling}

Computational fluid dynamic (CFD) analyses are conducted for the NACA0012 airfoil using the HYDRA code. HYDRA $[18]$ is an unstructured finite volume solver of the compressible Reynolds averaged Navier Stokes equations. The SpalartAllmaras turbulence model has been employed to solve viscous, Navier-Stokes equations.

\subsection{Computational domain and boundary conditions}

The domain size plays an important role in prediction of accurate aerodynamic forces [19]. Considering Vos' study, the domain used here extends 20 chord upstream, above and below the airfoil and 30 chord downstream as shown in Figure 1.

A C type topology has been used in order to generate the structured grid. In order to check mesh dependency of the solution, a number of increasingly fine meshes were used. The lift and drag coefficients were employed as performance parameters to determine the influence of grid size on the results. A final grid of 77665 mesh nodes with non-dimensional near wall mesh spacing
$Y^{+}<1$ was selected for further investigations. The lift and drag coefficients are in agreement with other published results showing less than $2 \%$ and $6 \%$ error as discussed in result section.

The airfoil boundary as shown in Figure 1 is treated as a wall with no slip conditions and all other boundaries have been considered as free stream boundaries. For the moving airfoil, the surface velocity is specified, and for the free stream boundary Mach number, total pressure and density have been specified.

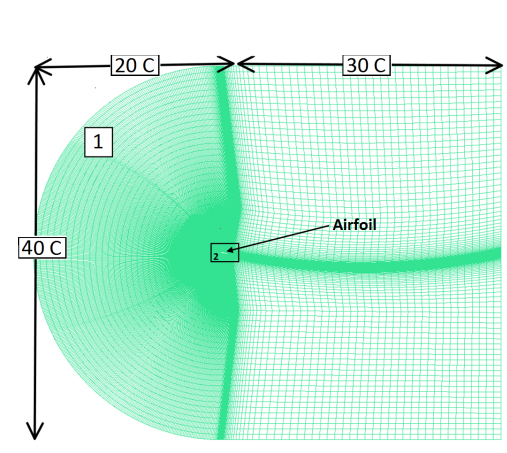

(a) Domain size

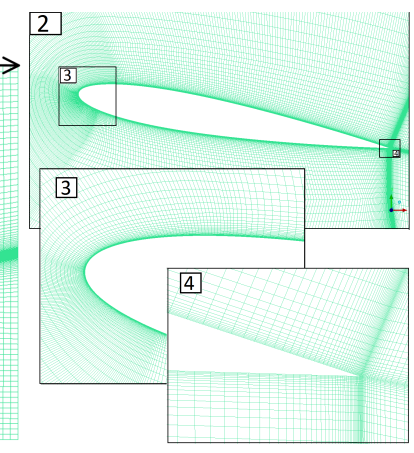

(b) Near View
FIGURE 1: Computational CFD mesh

\subsection{Moving mesh algorithm}

HYDRA has previously been developed to solve for moving mesh problems. Suppose domain D with its bounding surface $\mathrm{B}$ at time step $\mathrm{t}$ is a valid CFD mesh domain as shown in Figure 2. The domain $D^{\prime}$ and corresponding bounding surface $S^{\prime}$ is the deformed mesh after a time interval dt. This deformed mesh domain should be computationally valid with minimal distortion of the grid cells. A deforming mesh technique given by Amirante et al. [20] is used to generate the new grid based on the input received. In this method, a local stiffness matrix $\left[K^{H}\right]$ has to be defined. The choice of stiffness has a strong impact on the construction of the deformed mesh. For the current work, an elastic analogy has been considered in which construction of the stiffness matrix is similar to classical structural analysis. The displacement vector $\mathrm{q}$ in the element modelled as a combination of shape function and nodal values:

$$
q(x, y, z)=[N] q^{H}
$$

where $[\mathrm{N}]$ is the shape function and $\mathrm{q}$ is a function of position $\mathrm{r}=(\mathrm{x}, \mathrm{y}, \mathrm{z}) ; q^{H}$ denotes the local displacement of element $\mathrm{H}$. The relationship between strain $(\varepsilon)$ and displacement for a generic 

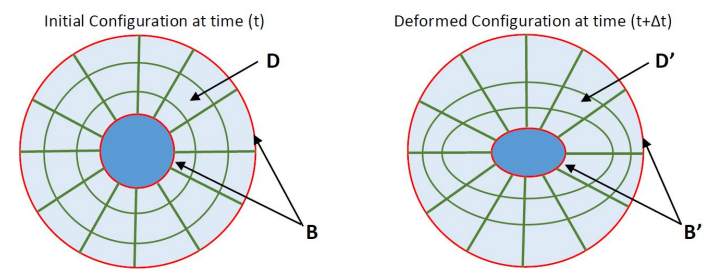

FIGURE 2: Initial and deformed mesh configurations

element $\mathrm{H}$ can be expressed as:

$$
\varepsilon=[D] q=[D][N] q^{H}=[\beta] q^{H}
$$

where [D] is a matrix of differential operators which represents the relation of strain to the displacement using linear elastic theory. For the elastic material, stress $(\sigma)$ can be defined using Hooke's law:

$$
\sigma=[E] \varepsilon
$$

in which [E] is the elastic matrix which is only function of Young's modulus and poison's ratio. Further, an energy balance for the element $\mathrm{H}$ can be written as :

$$
\begin{gathered}
q^{H^{T}} F^{H}=\int \varepsilon^{T} \sigma d V \\
q^{H^{T}} F^{H}=\int\left([\beta] q^{H}\right)^{T}[E][\beta] q^{H} d V
\end{gathered}
$$

Matrix formulation of Hooke's law relates the stress and strain tensor as follow:

$$
[\tau]=(\lambda+2 G / 3)(\nabla . u) I+2 G[\varepsilon]^{\circ}
$$

where $G$ and $\lambda$, are the first and second Lame constants. The original shape and volume of element are weighted by $2 \mathrm{G}$ and $\lambda+2 G / 3$.

$$
2 G=\alpha_{s}\left|\phi_{s}\right| ; \lambda+\frac{2 G}{3}=\alpha_{v}\left|\phi_{v}\right|
$$

$\alpha_{s}$ and $\alpha_{v}$ are user input parameters controlling element shape and volume. The quality of the mesh is sensitive to these param- eters which must be chosen with care.

\subsection{Unsteady flow simulation method}

Previous applications of the moving mesh technique in HYDRA have assumed quasi steady flow. This study includes the time accurate simulation over a moving airfoil. It extends the testing and validation of the moving mesh technique [20] to high amplitude pitching airfoil test cases. A flow chart for the numerical subroutine used is shown in Figure 3. Flow has been simulated at an initial time step and the airfoil profile is updated using a subroutine to generate the coordinates of the new position, since the position of the airfoil changes with respect to time. The new CFD grid is generated for an updated airfoil boundary based on the elastic analogy explained previously. The mesh motion has been taken into consideration in the convection term of the Arbitrary Lagrangian Eulerian (ALE) integral form of the conservation equation. The dynamic stall simulation took 5 days running on $24 \mathrm{CPUs}$ of a $\mathrm{PC}$ cluster. The mesh adaptation took approximately $30 \%$ of the whole computational time.

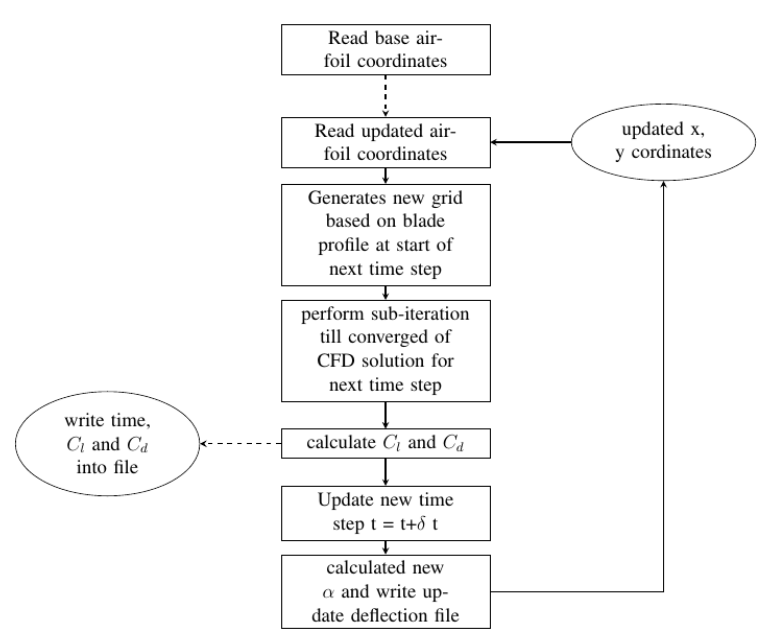

FIGURE 3: Flowchart of subroutine used in HYDRA source code

\section{Results}

CFD results are presented in the following subsections in terms of lift coefficient and drag coefficient plots and velocity and pressure contours.

\subsection{Steady state solutions}

The lift coefficient of a static airfoil increases with increase in angle of attack until it reaches its maximum value. A drop in lift curve slope occurs close to this point because of onset of flow 
TABLE 1: Lift and drag coefficient at 10 degree angle of attack

\begin{tabular}{|c|c|c|c|}
\hline & HYDRA(Present) & CFD [22] & Error \\
\hline$C_{l}$ & 1.07911 & 1.08 to 1.10 & $1.93 \%$ \\
\hline$C_{d}$ & 0.0136 & 0.0123 to 0.0129 & $5.14 \%$ \\
\hline
\end{tabular}

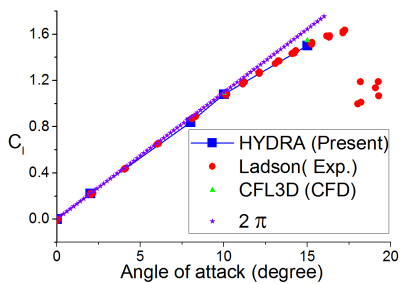

(a) Lift coefficient vs angle of attack

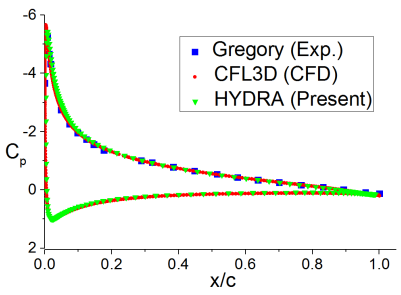

(c) Pressure coefficient at 10 degree angle of attack

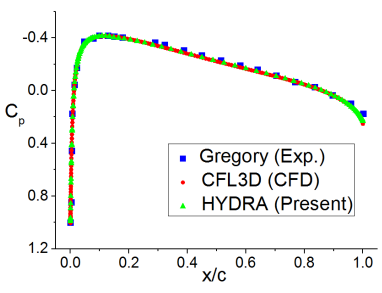

(b) Pressure coefficient at 0 degree angle of attack

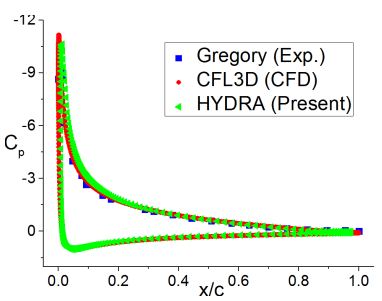

(d) Pressure coefficient at 15 degree angle of attack
FIGURE 4: Steady state pressure and lift coefficient plots at different angle of attacks

separation on the airfoil suction surface. The separation of flow becomes more significant with further increase in angle of attack and there is an angle at which flow becomes fully separated and beyond which drop in lift coefficient occurs. This angle is known as the static stall angle. The lift coefficient at this angle is the maximum lift coefficient.

Results presented are for a test case with Reynolds number $R e=6 \times 10^{6}$ and Mach number $M=0.15$. Lift coefficients from HYDRA at different angles of attack are compared with the experimental Ladson et al. [21] and CFD results obtained with CFL3D code [22] in Figure 4(a).

For a thin airfoil, at lower angle of attack the lift coefficient is given by $2 \Pi \alpha$, which is also plotted. Lift and drag coefficient at 10 degree angle of attack are tabulated and compared with CFD data from NASA [22] values in Table 1 . The difference is calculated with respect to largest value in the literature. $\left(C_{l}\right.$ [22]$\left.C_{l_{\text {HYDRA }}}\right) / C_{l_{\text {HYDRA }}}$ gives the difference in $C_{l}$ value. The pressure

coefficient at 0,10 and 15 degree angles of attack are plotted and compared with Gregory et al. [23] values in Figures 4 (b), (c) and (d) respectively. Present results from HYDRA are in good agreement with the other authors' computational as well as experimental steady state results for the NACA0012 airfoil.

\subsection{Dynamic Stall}

The unsteady aerodynamics of the NACA0012 airfoil subjected to a strong pitching motion are now considered. Dynamic stall is an unsteady aerodynamic phenomenon in which onset of stall occurs at an angle of attack much greater than the static stall angle. Also there is sudden drop in lift unlike the gradual drop in lift for the steady state case. In this section results are presented for an airfoil pitching about its quarter chord point with an amplitude of 10 degrees about the mean angle of attack of 15 degrees. Solutions for different Mach number, reduced frequency and Reynolds number are presented. The pitching motion can be expressed as

$$
\alpha(t)=\alpha_{o}+\alpha_{a} \sin (\omega t)
$$

\subsubsection{Comparison with previous work}

This section presents the results for a test case with Re of 1 million and reduced frequency of 0.1 . The lift and drag coefficients obtained are compared with experimental results published by McCroskey et al. [17] in Figure 5. During the upstroke of the pitching motion, the angle of incidence increases and the lift coefficient is in good agreement with experimental results. A maximum lift coefficient of 2.30 occurs at approx $22.2^{\circ}$, which is close to the experimental measurement as shown in Figure 5 (a). The overall trend of the drag coefficient is also similar to the experimental values shown in Figure 5 (b).

During the downstroke of the pitching airfoil, HYDRA under predicts the lift coefficient from incidence $24^{\circ}$ to $21^{\circ}$ and over predicts other angles of attack in the downstroke. The leading edge vortex separation takes place during upstroke motion and

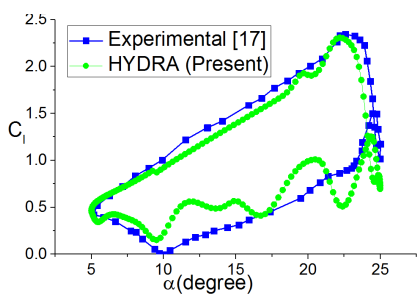

(a) $C_{l}$ comparison with experimental data

FIGURE 5: Lift and drag coefficient comparison with experimental results McCroskey et al. [17]

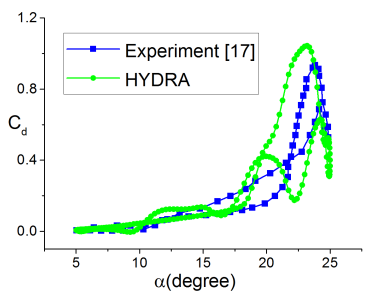

(b) $C_{d}$ comparison with experimental data 


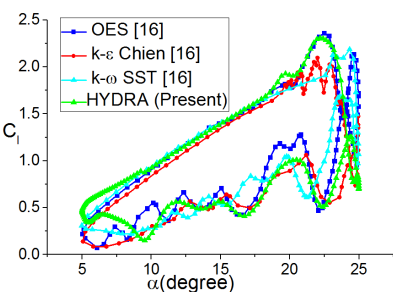

(a) $C_{l}$ comparison with computational data

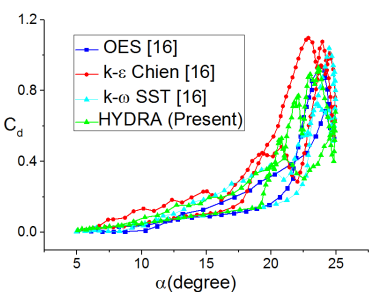

(b) $C_{d}$ comparison with computational data
FIGURE 6: Lift and drag coefficient comparison with computational results Martinat et al. [16]

secondary vortex separation takes place near to the end of upstroke and partially separation during the downstroke motion. This results in fully separated flow from $25^{\circ}$ to $9^{\circ}$ and the discrepancy in the CFD load prediction. In the experiments three dimensional effects could contribute to these differences in the fully separated flow region. HYDRA results are compared with other workers' computational results in Figure 6. It is observed that the HYDRA results are in reasonably agreement with the $\kappa-\varepsilon$ organised eddy simulation (OES) model presented by Martinat et al. [16]. During the upstroke, the maximum lift coefficient and drag coefficient are in much better agreement with the OES model. Also the angle at which $C_{l_{\max }}$ and $C_{d_{\max }}$ occur is in close proximity to the published OES model results. During the downstroke the overall trend of the lift coefficient obtained by HYDRA is quite similar to computational results from the OES model [16].

It is argued by Martinat et al. [16] that the fluctuations appearing in the lift and drag hysteresis loop are due to coherent vortex shedding. This vortex shedding may be more pronounced in the two-dimensional simulations then in the experiments, because of flow confinement induced by the two-dimensional approximation. It can be concluded the lift and drag coefficients obtained by HYDRA are both qualitatively and quantitatively in agreement with the published experiments and computational results.

\subsubsection{Effects of reduced frequency}

This sections presents HYDRA results at different reduced frequencies for fixed $R e$ of 2.5 million and Mach number 0.2. Figure 7 shows the lift and drag coefficient vs angle of attack at reduced frequencies of $0.1,0.15 \& 0.25$. It has been found in the present study that for an airfoil pitching with lower reduced frequency, the maximum lift coefficient and stall occur prior to the peak angle of attack of the cycle. Stall angle of attack increases with increase in reduced frequency due to delay of the separation of the leading edge vortex as explained in more detail later. The flow physics predicted is compared with that deduced by other workers' from experiments.

(I) $\mathrm{k}=\mathbf{0 . 2 5}$

Figures 8 and 9 show streamline patterns superimposed over

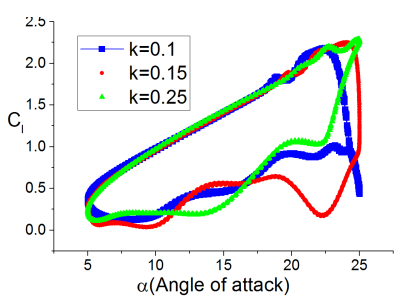

(a) $C_{l}$ vs $\alpha$

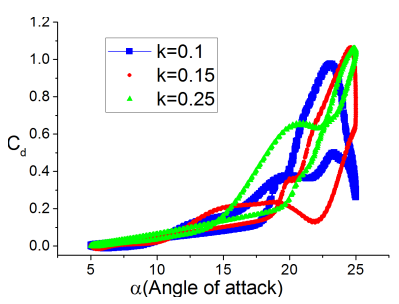

(b) $C_{d}$ vs $\alpha$
FIGURE 7: Lift and drag coefficient at different reduced frequen$\operatorname{cies}(\mathrm{k}=0.1,0.15 \& 0.25)$

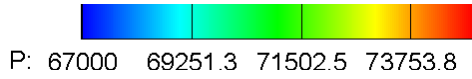

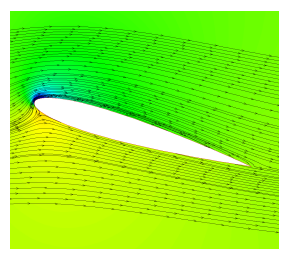

(a) $\alpha=15^{\circ}$ initial

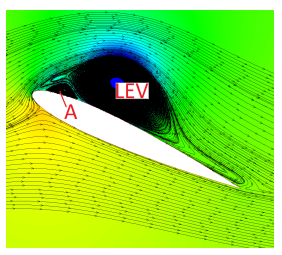

(d) $\alpha=24.2^{\circ}(\uparrow)$

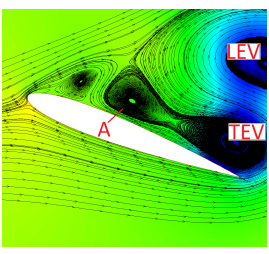

(g) $\alpha=19^{\circ}(\downarrow)$

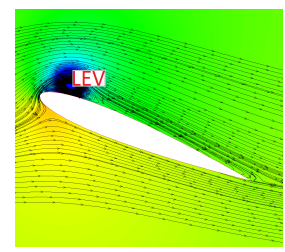

(b) $\alpha=19^{\circ}(\uparrow)$

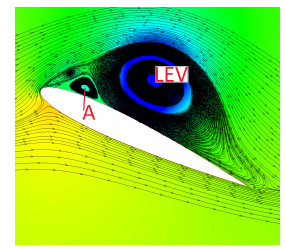

(e) $\alpha=25^{\circ}$ (peak)

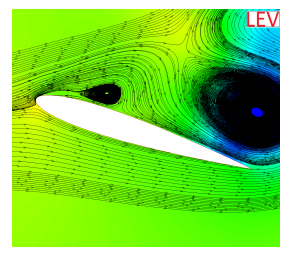

(h) $\alpha=17.4^{\circ}(\downarrow)$

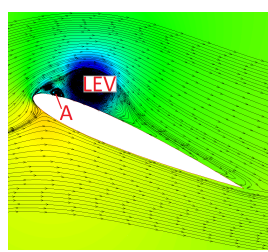

(c) $\alpha=21.8^{\circ}(\uparrow)$

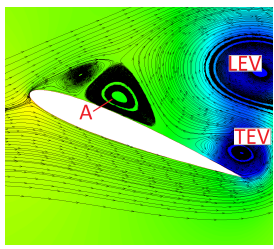

(f) $\alpha=19.8^{\circ}(\downarrow)$

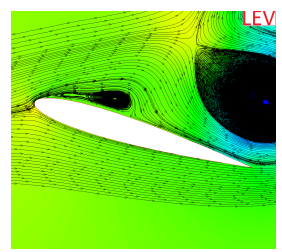

(i) $\alpha=15.8^{\circ}(\downarrow)$
FIGURE 8: Streamline pattern superimposed over pressure contour for $\mathrm{k}=0.25 ;(\downarrow)$ denotes downstroke, $(\uparrow)$ denotes upstroke

pressure and velocity contours respectively at different angle of attack positions for a reduced frequency $\kappa=0.25$.

\section{(a) Formation of LEV}

During upstroke motion, the flow is fully attached at $15^{\circ}$. As the angle of attack $(\alpha)$ increases, the lift coefficient also increases due to large suction at the leading edge associated with formation of a leading edge vortex (LEV) as shown in Figure 8 (b). Further, the LEV strengthens and covers half of the chord at an 


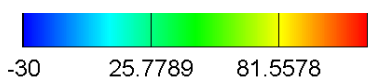

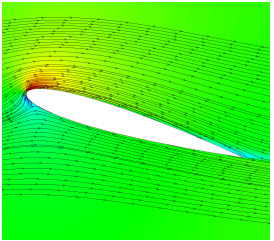

(a) $\alpha=15^{\circ}$ initial

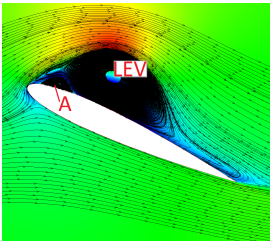

(d) $\alpha=24.2^{\circ}(\uparrow)$

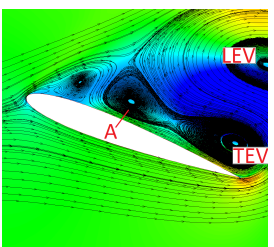

(g) $\alpha=19^{\circ}(\downarrow)$

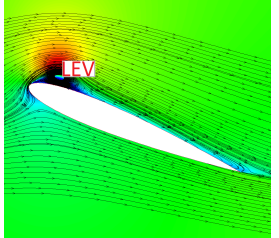

(b) $\alpha=19^{\circ}(\uparrow)$

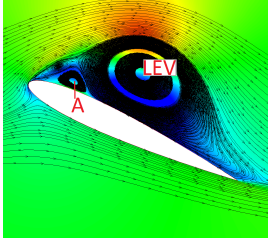

(e) $\alpha=25^{\circ}$ (peak)

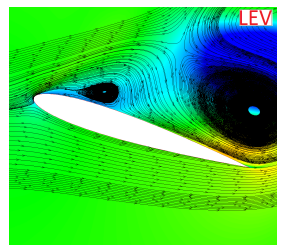

(h) $\alpha=17.4^{\circ}(\downarrow)$

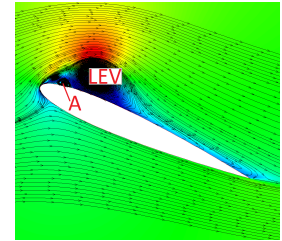

(c) $\alpha=21.8^{\circ}(\uparrow)$

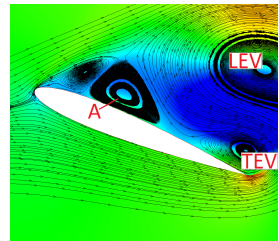

(f) $\alpha=19.8^{\circ}(\downarrow)$

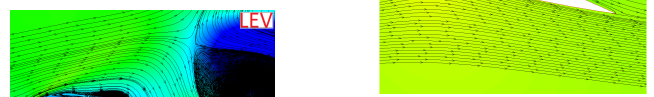

(a) $\alpha=15^{\circ}$ ( $)$

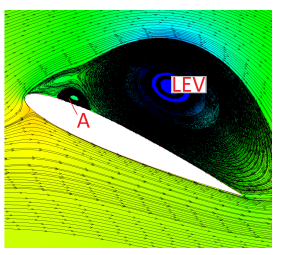

(d) $\alpha=21.4^{\circ}(\uparrow)$

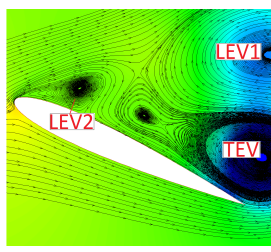

(g) $\alpha=23^{\circ}(\downarrow)$

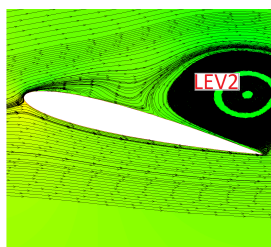

(j) $\alpha=13.8^{\circ}(\downarrow)$

\section{P: $67000 \quad 69251.3 \quad 71502.5 \quad 73753.8$}

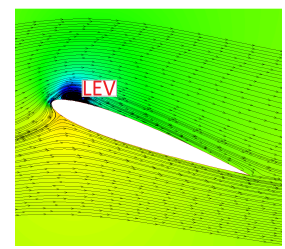

(b) $\alpha=17.8^{\circ}(\uparrow)$

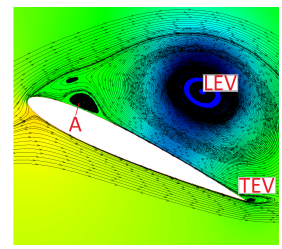

(e) $\alpha=23.8^{\circ}(\uparrow)$

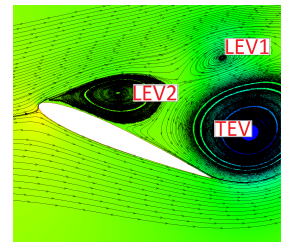

(h) $\alpha=21.8^{\circ}(\downarrow)$

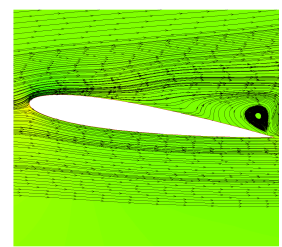

(k) $\alpha=10.2^{\circ}(\downarrow)$

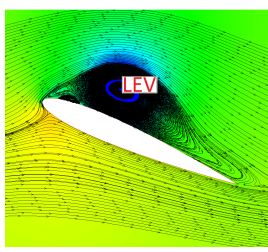

(c) $\alpha=19.8^{\circ}(\uparrow)$

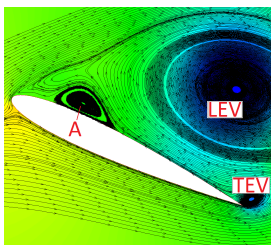

(f) $\alpha=25^{\circ}$ (peak)

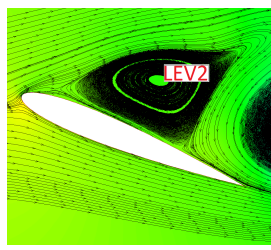

(i) $\alpha=19.8^{\circ}(\downarrow)$

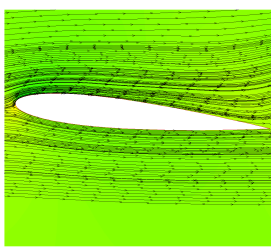

(1) $\alpha=12.2^{\circ}(\uparrow)$ moves towards the trailing edge as shown in Figure $8(\mathrm{a}-\mathrm{e})$. The trailing edge vortex (TEV) also includes low pressure and is in direct contact with airfoil surface as shown in Figure 8 (f) . Vortex A and the TEV both have anticlockwise rotation, their merger forms a bigger TEV. This TEV pushes the primary LEV further downstream during downstroke motion.

In summary, it has been observed that for higher reduced frequency, the formation of a primary LEV takes place during
FIGURE 10: Streamline pattern superimposed over pressure contour for $\mathrm{k}=0.15 ;(\downarrow)$ denotes downstroke, $(\uparrow)$ denotes upstroke

\section{(a) Formation of LEV}

At the initial angle of attack, $15^{\circ}$, the flow is fully attached. As 


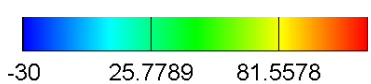

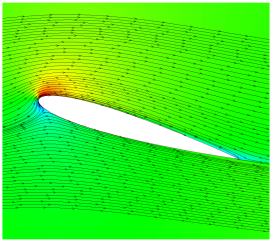

(a) $\alpha=15^{\circ}(\uparrow)$

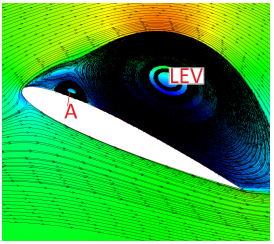

(d) $\alpha=21.4^{\circ}(\uparrow)$

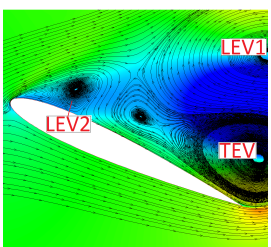

(g) $\alpha=23^{\circ}(\downarrow)$

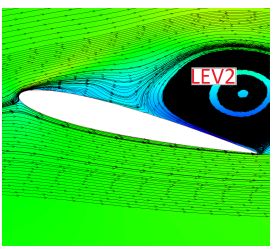

(j) $\alpha=13.8^{\circ}(\downarrow)$

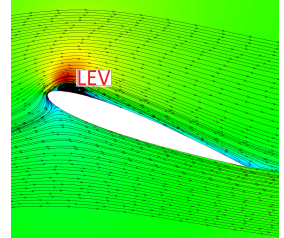

(b) $\alpha=17.8^{\circ}(\uparrow)$

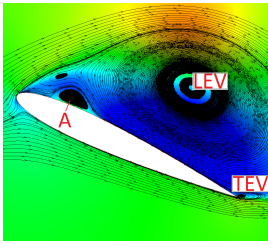

(e) $\alpha=23.8^{\circ}(\uparrow)$

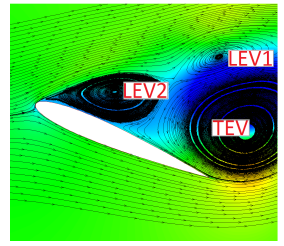

(h) $\alpha=21.8^{\circ}(\downarrow)$

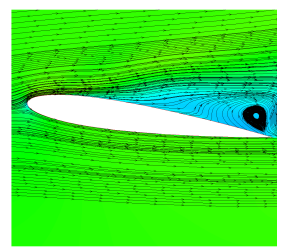

(k) $\alpha=10.2^{\circ}(\downarrow)$

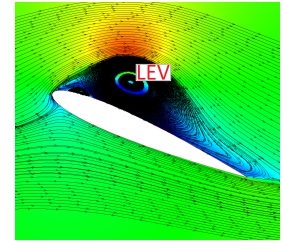

(c) $\alpha=19.8^{\circ}(\uparrow)$

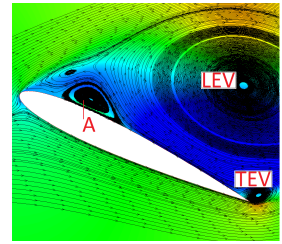

(f) $\alpha=25^{\circ}$ (peak)

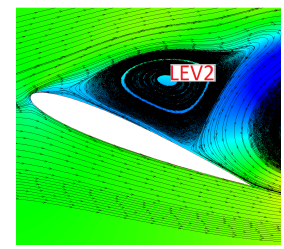

(i) $\alpha=19.8^{\circ}(\downarrow)$

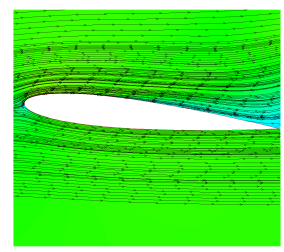

(1) $\alpha=12.2^{\circ}(\uparrow)$
FIGURE 11: Streamline pattern superimposed over velocity contour for $\mathrm{k}=0.15 ;(\downarrow)$ denotes downstroke, $(\uparrow)$ denotes upstroke

the angle of attack $(\alpha)$ increases, a suction peak occurs near the leading edge surface which later on plays an important role in the formation of the LEV. LEV formation takes place at $18^{\circ}$ as shown in Figure 10 (b). The size and strength of the LEV increases with increase with $\alpha$, which results in higher differences in the pressure on the upper surface (suction surface) to the lower surface ( pressure surface ) and hence the higher lift. A further increase in $\alpha$ leads to separation of the LEV from the suction surface and the shed vortex propagates downstream. There is also formation of a trailing edge vortex (TEV) which grows in size as it merges with a small vortex A and pushes the primary LEV further downstream as shown in Figure 10 ( e - h ). With separation of the LEV, the lift coefficient drops suddenly as shown in Figure 7

\section{(b) Formation and shedding of secondary LEV}

Formation of the secondary LEV occurs during the transition from upstroke to downstroke motion. Figure 10 (g-i) shows the growth of secondary LEV during the downstroke motion from $23^{\circ}$ to $19.8^{\circ}$, which causes a rise in lift. This secondary vortex moves downstream during the remaining downstroke motion. It can be noted that the formation and shedding of the LEV takes place during upstroke motion, The secondary LEV occurs during transition, and shedding occurs in the downstroke motion for the moderate reduced frequency.

\section{(III) $\mathrm{k}=\mathbf{0 . 1 0}$}

Figures 12 and 13 show streamline patterns superimposed over pressure and velocity contours for a reduced frequency of 0.10 .

\section{(a) Formation and Shedding of LEV}

During the upstroke flow remains attached to the surface at $15^{\circ}$, A sharp drop in pressure occurs near the leading edge with further increase in angle of attack and this marks the formation of a leading edge vortex (LEV) at $17^{\circ}$. With further increase in angle of attack, the LEV grows and covers almost half of the suction surface at $18.2^{\circ}$. Later on, the separation of primary LEV takes place at $22.6^{\circ}$ and lift drops abruptly as shown in Figure 12 (e) and 7 .

\section{(b) Formation and shedding of secondary LEV}

The secondary LEV formation also takes place in the upstroke motion. The separated primary LEV moves downstream. The vortex A also merges with the TEV and results in a strengthened TEV. This TEV breaks the primary vortex and pushes the LEV further downstream. Part of the primary vortex merges with the secondary LEV on the suction surface as shown in Figure 12 (g h) and this results in the gain of lift during downstroke from $25^{\circ}$ to $19.8^{\circ}$. Finally, the secondary vortex separates and propagates downstream during the remaining downstroke motion.

\subsubsection{Summary of vortex shedding}

McCroskey et al. [1] have also categorized the formation of the primary LEV, secondary LEV and their shedding into three types. At higher reduced frequencies, the separation of LEV and propogation and separation of secondary LEV takes place in downstroke. For moderate reduced frequency, the shedding of primary LEV could takes place in upstroke and for lower reduced frequency the shedding of secondary vortex also could be observed in upstroke motion itself. A similar flow physics also found in their experimental study by Sharma and Poddar [24]. The present HYDRA code results are also in agreement with the above finding.

\section{Conclusion}

The HYDRA CFD code has been developed and evaluated for simulation of blade flow with high amplitude pitching. Results have been compared with previously published experimental and computational results. It has been concluded that during upstroke 


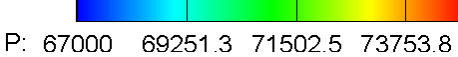

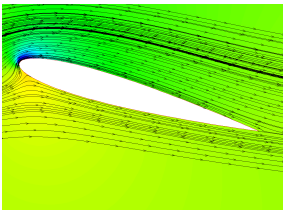

(a) $\alpha=15^{\circ}(\uparrow)$

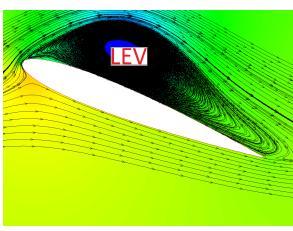

(d) $\alpha=19^{\circ}(\uparrow)$

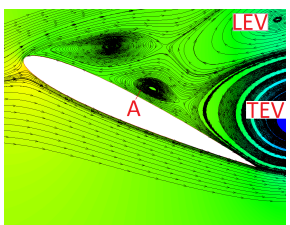

(g) $\alpha=25^{\circ}$ (peak)

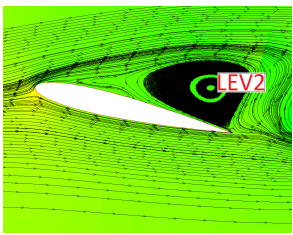

(j) $\alpha=13.8^{\circ}(\downarrow)$

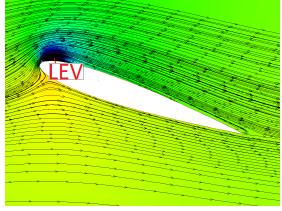

(b) $\alpha=17^{\circ}(\uparrow)$

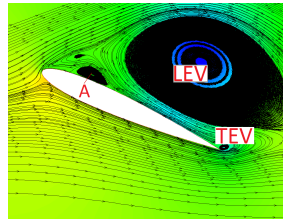

(e) $\alpha=22.6^{\circ}(\uparrow)$

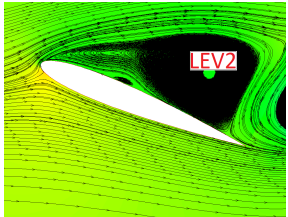

(h) $\alpha=19.8^{\circ}(\downarrow)$

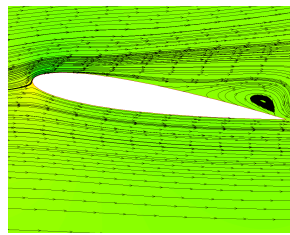

(k) $\alpha=10.6^{\circ}(\downarrow)$

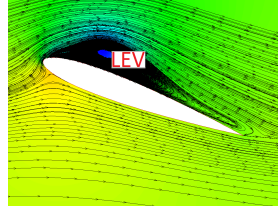

(c) $\alpha=18.2^{\circ}(\uparrow)$

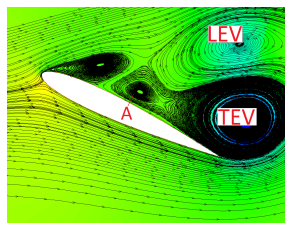

(f) $\alpha=24.6^{\circ}(\uparrow)$

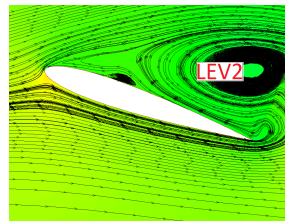

(i) $\alpha=17.4^{\circ}(\downarrow)$

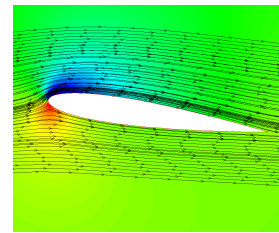

(1) $\alpha=9.8^{\circ}(\uparrow)$
FIGURE 12: Streamline pattern superimposed over pressure contour for $\mathrm{k}=0.10 ;(\downarrow)$ denotes downstroke, $(\uparrow)$ denotes upstroke

motion, HYDRA results are in good agreement with experimental and computational results. There is some undulation in lift and drag and differences with experimental during the downstroke motion. However these differences could be associated with the approximation of three dimensional flow to the two dimensional flow. There are also some differences in the results during downstroke, with other workers' computational published results and these difference could be associated with the different turbulence models used. The previously published computational results also have undulation of lift during downstroke and there is variation in results due to the different turbulent models. Two dimensional dynamic stall results are expected to give insight into flow at the mid-span of the blade or wing. However, it should be noted that separated flow intrinsically unsteady and three dimensional. Three dimensional modelling clearly required to model the wing tip and wing let locations. The present method

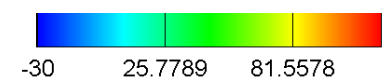

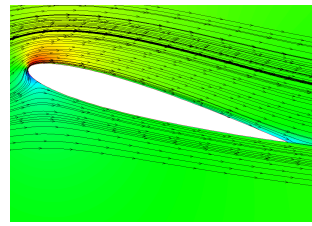

(a) $\alpha=15^{\circ}(\uparrow)$

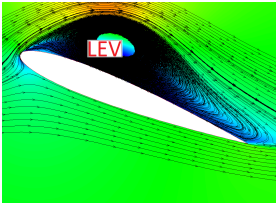

(d) $\alpha=19^{\circ}(\uparrow)$

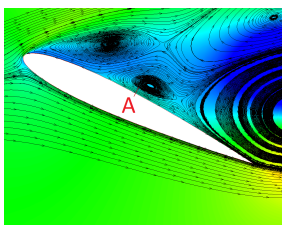

(g) $\alpha=25^{\circ}$ (peak)

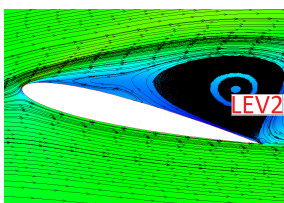

(j) $\alpha=13.8^{\circ}(\downarrow)$

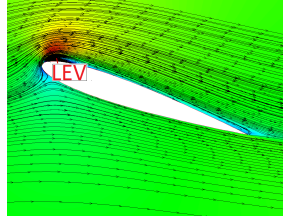

(b) $\alpha=17^{\circ}(\uparrow)$

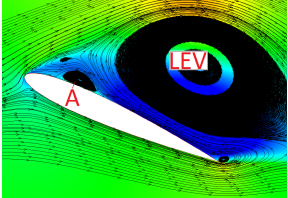

(e) $\alpha=22.6^{\circ}(\uparrow)$

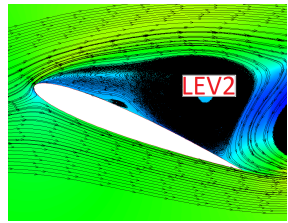

(h) $\alpha=19.8^{\circ}(\downarrow)$

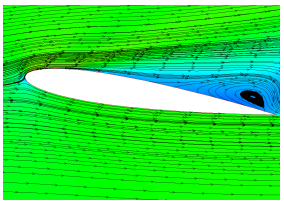

(k) $\alpha=10.6^{\circ}(\downarrow)$

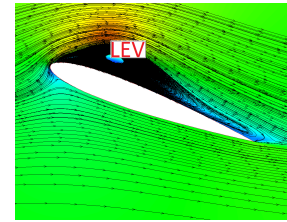

(c) $\alpha=18.2^{\circ}(\uparrow)$

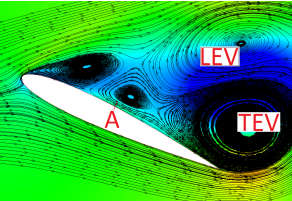

(f) $\alpha=24.6^{\circ}(\uparrow)$

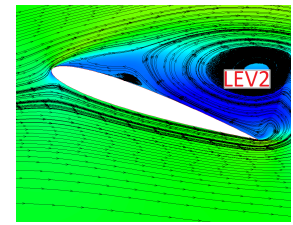

(i) $\alpha=17.4^{\circ}(\downarrow)$

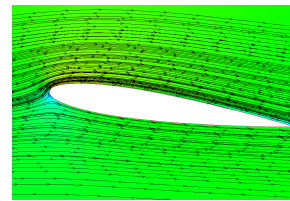

(1) $\alpha=9.8^{\circ}(\uparrow)$
FIGURE 13: Streamline pattern superimposed over velocity contour for $\mathrm{k}=0.10 ;(\downarrow)$ denotes downstroke, $(\uparrow)$ denotes upstroke

can be relatively straight forward extended to 3D but the computing requirements are a restricting factor.

Effects of reduced frequency were also studied. It has been concluded that at higher reduced frequency the formation of the LEV takes place during the upstroke, while shedding of LEV, formation and shedding of secondary LEV takes place during the downstroke. However, for moderate reduced frequency, the shedding of the LEV takes place in the upstroke itself and further for lower reduced frequency, even the secondary LEV formation takes place during upstroke motion. Similar physics have been deduced from experiments by McCroskey et al. [1], and Sharma and Poddar [24] as mentioned previously. Hence, it is concluded that HYDRA is able to capture the overall trend of hysteresis loop of the lift coefficient and drag coefficient and it is also able to predict the stall angle and formation and shedding of vortices 
in reasonably good agreement with available experimental data. Trends observed with varying reduced frequency are also consistent with experiment.

\section{REFERENCES}

[1] McCroskey, W., Carr, L., and McAlister, K., 1976. "Dynamic stall experiments on oscillating airfoils". AIAA Journal, 14(1), pp. 57-63.

[2] Mehta, U. B., 1978. "Dynamic stall of an oscillating airfoil". 1978. 32.

[3] Fung, K.-Y., and Carr, L., 1991. "Effects of compressibility on dynamic stall”. AIAA journal, 29(2), pp. 306-308.

[4] Currier, J. M., and Fung, K.-Y., 1992. "Analysis of the onset of dynamic stall”. AIAA journal, 30(10), pp. 2469-2477.

[5] Ekaterinaris, J. A., and Platzer, M. F., 1998. "Computational prediction of airfoil dynamic stall". Progress in aerospace sciences, 33(11), pp. 759-846.

[6] Raffel, M., Kompenhans, J., and Wernert, P., 1995. "Investigation of the unsteady flow velocity field above an airfoil pitching under deep dynamic stall conditions". Experiments in Fluids, 19(2), pp. 103-111.

[7] Wang, S., Ingham, D. B., Ma, L., Pourkashanian, M., and Tao, Z., 2010. "Numerical investigations on dynamic stall of low Reynolds number flow around oscillating airfoils". Computers \& Fluids, 39(9), pp. 1529-1541.

[8] Leishman, J., 1990. "Dynamic stall experiments on the naca 23012 aerofoil". Experiments in Fluids, 9(1-2), pp. 49-58.

[9] Leu, T. S., Yu, J., Hu, C., Miau, J., Liang, S., Li, J., Cheng, J., and Chen, S., 2012. "Experimental study of free stream turbulent effects on dynamic stall of pitching airfoil by using particle image velocimetry". In Applied Mechanics and Materials, Vol. 225, Trans Tech Publ, pp. 103-108.

[10] Leishman, J. G., 2006. Principles of Helicopter Aerodynamics with CD Extra. Cambridge university press.

[11] Sarkar, S., and Venkatraman, K., 2008. "Influence of pitching angle of incidence on the dynamic stall behavior of a symmetric airfoil". European Journal of MechanicsB/Fluids, 27(3), pp. 219-238.

[12] Akbari, M., and Price, S., 2003. "Simulation of dynamic stall for a naca 0012 airfoil using a vortex method". Journal of fluids and structures, 17(6), pp. 855-874.

[13] Kang, C.-k., Aono, H., Trizila, P., Baik, Y., Rausch, J. M., Bernal, L., Ol, M. V., and Shyy, W., 2009. "Modeling of pitching and plunging airfoils at Reynolds number between $1 \times 10^{4}$ and $6 \times 10^{4}$ ". In 27th AIAA Applied Aerodynamics Conference, pp. 22-25.

[14] Rival, D., and Tropea, C., 2010. "Characteristics of pitching and plunging airfoils under dynamic-stall conditions". Journal of Aircraft, 47(1), pp. 80-86.

[15] Wernert, P., Geissler, W., Raffel, M., and Kompenhans, J.,
1996. "Experimental and numerical investigations of dynamic stall on a pitching airfoil". AIAA journal, 34(5), pp. 982-989.

[16] Martinat, G., Hoarau, Y., Braza, M., Vos, J., and Harran, G., 2008. "Numerical simulation of the dynamic stall of a naca 0012 airfoil using des and advanced oes/urans modelling". In Advances in Hybrid RANS-LES Modelling. Springer, pp. 271-278.

[17] McCroskey, W., Mcalister, K. W., Carr, L., and Pucci, S., 1982. "An experimental study of dynamic stall on advanced airfoil sections. volume 1: Summary of the experiment".

[18] Moinier, P., 1999. "Algorithm developments for an unstructured viscous flow solver". PhD thesis, Oxford University.

[19] Vos, W. W., 2006. "The verification and validation of preliminary CFD results for the construction of an a priori aerodynamic model.".

[20] Amirante, D., Hills, N., and Barnes, C., 2012. "A moving mesh algorithm for aero-thermo-mechanical modelling in turbomachinery". International Journal for Numerical Methods in Fluids, 70(9), pp. 1118-1138.

[21] Ladson et al. experimental data 2D NACA 0012 Airfoil Validation, https://turbmodels.larc.nasa. gov/naca0012_validation/clcd_ladson_ expdata.dat.

[22] CFD results from NASA website,2D NACA 0012 Airfoil Validation, http://turbmodels.larc.nasa. gov/naca0012_val_sa.html.

[23] Gregory, N., and O'reilly, C., 1973. Low-Speed aerodynamic characteristics of NACA 0012 aerofoil section, including the effects of upper-surface roughness simulating hoar frost. HM Stationery Office London.

[24] Sharma, D., and Poddar, K., 2013. "Investigation of dynamic stall characteristics for flow past an oscillating airfoil at various reduced frequencies by simultaneous piv and surface pressure measurements". In Proceedings of PIV 13: 10th International Symposium on Particle Image Velocimetry, Delft, The Netherlands, July. 